\title{
Liquid nickel salts: synthesis, crystal structure determination and electrochemical synthesis of nickel nanoparticles
}

\author{
$\underline{\text { Jeroen Sniekers }}^{1}$, Ken Verguts ${ }^{1}$, Neil R. Brooks ${ }^{1}$, Stijn Schaltin ${ }^{2}$, Thanh Hai Phan ${ }^{1}$, Thi Mien Trung \\ Huynh $^{1}$, Luc Van Meervelt ${ }^{1}$, Steven De Feyter ${ }^{1}$, Jin Won $\mathrm{Seo}^{2}$, Jan Fransaer ${ }^{2}$, Koen Binnemans ${ }^{1}$ \\ ${ }^{1}$ KU Leuven, Department of Chemistry, Celestijnanlaan 200F - P.O. Box 2404, B-3001 Heverlee, \\ Belgium \\ ${ }^{2}$ KU Leuven, Department of Metallurgy and Materials Engineering, Kasteelpark Arenberg 44 - \\ P.O. Box 2450, B-3001 Heverlee, Belgium \\ E-mail: jeroen.sniekers@chem.kuleuven.be
}

Liquid metal salts are ionic liquids in which a redox active metal is incorporated into the cation of the ionic liquid. This way, very high metal concentrations are obtained. Liquid metal salts are excellent electrolytes for the electrodeposition of metals. Because the metal is incorporated into the cation, it will electromigrate towards the cathode during deposition, allowing a better mass transport to counteract the higher viscosity of the ionic liquid compared to a watery solution. Since the cathodic reaction is the deposition of metal, there are no issues with the cathodic decomposition of the ionic liquid components.

In this work, new nickel(II)-containing ionic liquids were synthesized, characterized and their electrochemistry was investigated. In these liquid metal salts, the nickel(II) cation is octahedrally coordinated by six $\mathrm{N}$-alkylimidazole ligands. The different counter anions that were used are bis(trifluoromethanesulfonyl)imide $\left(\mathrm{Tf}_{2} \mathrm{~N}^{-}\right)$, trifluoromethanesulfonate (OTf) and methanesulfonate $\left(\mathrm{OMs}^{-}\right)$. Several different $N$-alkylimidazoles were considered, with the alkyl side chain ranging in length from methyl to dodecyl. The newly synthesized liquid metal salts were characterized by CHN analysis, FTIR, DSC, TGA and viscosity measurements. An odd-even effect was observed for the melting temperatures and viscosities of the ionic liquids, with the complexes with an even number of carbon atoms in the alkyl chain of the imidazole having a higher melting temperature and a lower viscosity than the complexes with an odd number of carbons. The crystal structures of six of the nickel(II) complexes that are not liquid at room temperature were determined.

The electrochemistry of the compound with the lowest viscosity, $\left[\mathrm{Ni}(\mathrm{HeIm})_{6}\right]\left[\mathrm{Tf}_{2} \mathrm{~N}\right]_{2}$, was investigated. It was found that the nickel(II) cation could be reduced, but surprisingly no nickel deposits were obtained on the electrode. Instead, nickel nanoparticles were formed at $100 \%$ selectivity, as was confirmed by Transmission Electron Microscopy (TEM) measurements. The nickel nanoparticles were found to be superparamagnetic, as was investigated by a SQUID measurement. A mechanism for this nanoparticle formation was formulated, based on an extensive literature research and Scanning Tunneling Microscopy (STM) measurements. 